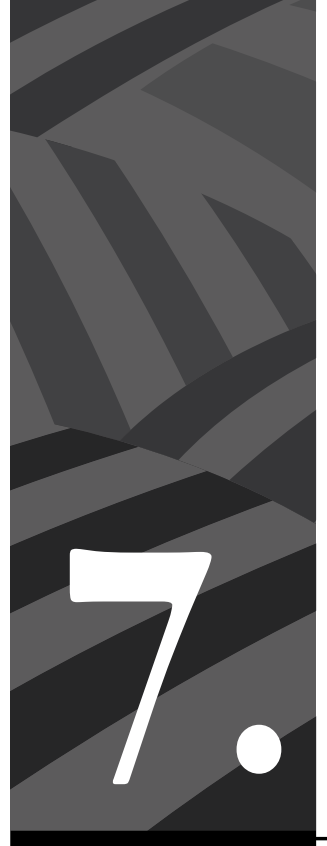

16 de mayo de 1984: pasado y presente en disputa. Algunas reflexiones a propósito del aniversario 35 de los acontecimientos 


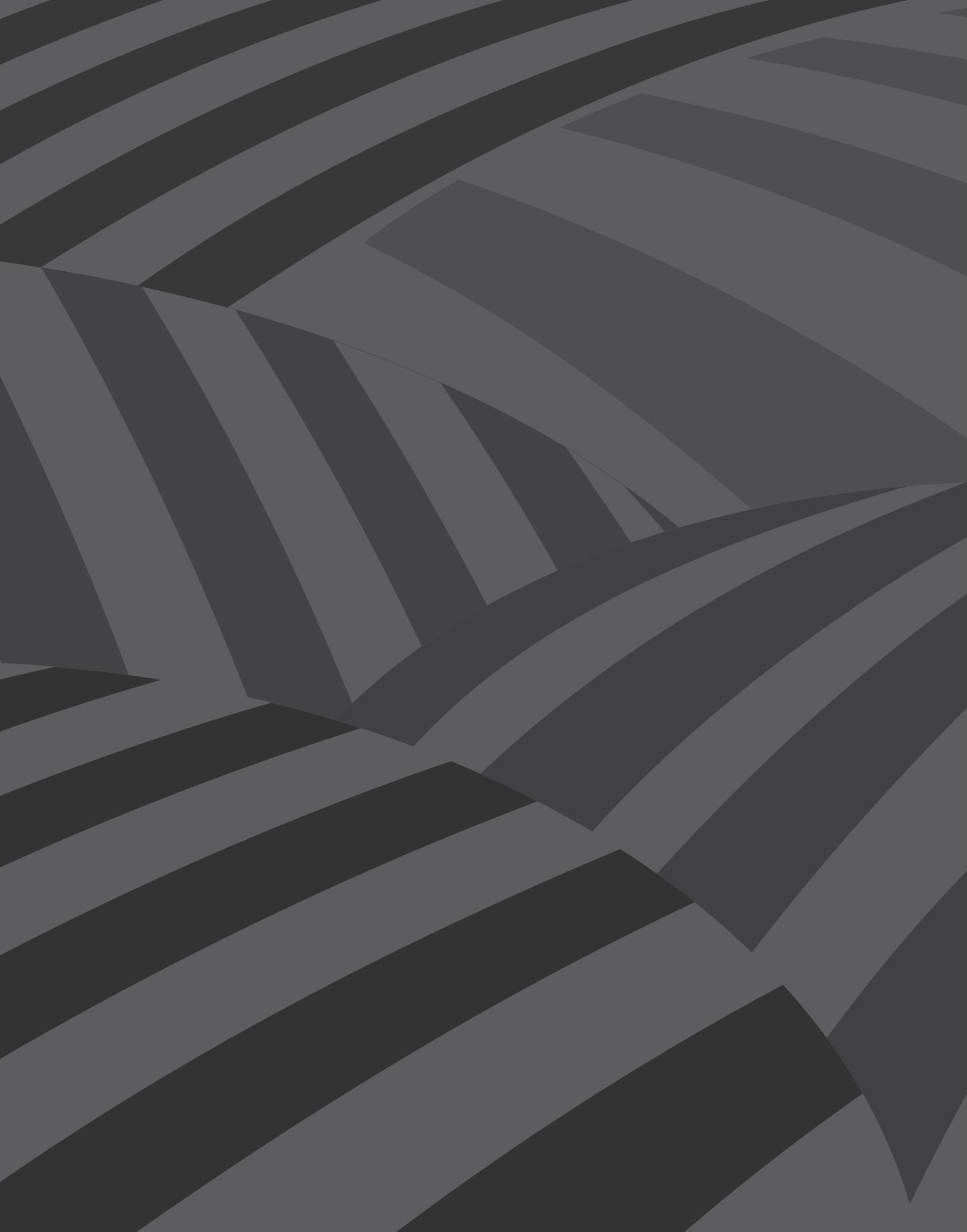




\section{6 de mayo de 1984: pasado y presente en disputa. Algunas reflexiones a propósito del aniversario 35 de los acontecimientos}

Por Juan Sebastián Flórez Herrera*

Resumen: Las memorias de los acontecimientos violentos del 16 de mayo de 1984 en la Universidad Nacional de Colombia han sido un tema de debate y disputa recurrente. El estudiantado nombró lo ocurrido como una masacre y denunció desapariciones forzadas masivas, mientras que otros sectores negaron o desestimaron estas afectaciones, se centraron en la violencia ejercida por las y los universitarios y los instigaron a probar sus denuncias. Este artículo aborda la relación problemática entre el negacionismo y las denuncias de las víctimas, en relación con las posibilidades de construir relatos esclarecedores. En su desarrollo, se pudo constatar que las posturas negacionistas ignoran o evaden hechos que permiten comprender este suceso como un caso de victimización del estudiantado.

Palabras clave: 16 de mayo, estudiantes, víctimas, prensa, verdad.

\section{May 16, 1984: A Fight for Past and Present. Reflections on the Occasion of the 35th Anniversary of the Events}

Abstract: The violent happenings and memories from May the 16th of 1984 at Universidad Nacional de Colombia have been recurrent topic of discussion. The student body named the happening as a massacre, and denounced massive forced disappearances, while other sectors denied or dismissed this affectations focusing on the violence exerted by the students and demanded them to prove their denounces. This text deals with the troublesome relation between negationism and the victims' memories, in relation to the possibilities of building enlightening tales that admit the sense of its experience. In its development, it could be seen that the denialist postures ignore or evade facts that allow it to understand this as a victimization case against the student body.

Keywords: May the $16^{\text {th }}$, students, victims, press, truth.

* Historiador de la Universidad Nacional de Colombia. Estudiante de la Maestría en Historia y Memoria de la Universidad Nacional de la Plata, Argentina. Miembro del Centro de Estudios Sociales y Culturales de la Memoria (CESYCME) y de su proyecto de investigación Archivos del Búho, Memoria Viva del Movimiento Estudiantil. Correo: jsflorezh@unal.edu.co 
Cómo citar este artículo: Flórez Herrera, Juan Sebastián (2019). 16 de mayo de 1984: pasado y presente en disputa. Algunas reflexiones a propósito del aniversario 35 de los acontecimientos. Revista Controversia, 213, 265-295.

Fecha de recepción: 20 de marzo de 2019

Fecha de aprobación: 4 de junio de 2019

\section{Introducción}

No es solo el pasado el que tiene un carácter indeterminado. El presente también se convierte en el lugar en el cual los elementos del pasado que fueron rechazados - en el sentido que no fueron integrados en una comprensión estable del pasado-, pueden repentinamente asediar el mundo con la misma insistencia y obstinación con que lo real agujerea lo simbólico.

Ortega 2008, p. 33

Las luchas por el derecho a la educación en Colombia —como el conjunto de las luchas de los movimientos sociales - tienen un importante auge en la segunda mitad del siglo xx. Las transformaciones políticas y sociales tanto de la sociedad colombiana como del propio mundo universitario decantaron en una conflictividad en ascenso que, no en pocos casos, cobró la vida y la seguridad de las y los universitarios. Como parte de esta historia, en el caso de la Universidad Nacional-Sede Bogotá (UN), las memorias del 16 de mayo de 1984 tienen un carácter emblemático y ocupan un lugar importante en las agendas de conmemoración del Movimiento Estudiantil.

El inicio de esta historia se fija comúnmente el 9 de mayo, cuando Jesús «Chucho» León, estudiante nariñense de sexto semestre de Odontología, fue detenido, torturado y asesinado en la ciudad de Cali. Su cuerpo -en lo que constituye una acción con alta dimensión enunciativa - fue abandonado en terrenos aledaños a la Universidad del Valle, donde fue encontrado en la mañana del 10 de mayo. Chucho León era dirigente de Cooperación Estudiantil, proceso gremial-institucional que 
lideró por estos años las principales luchas que tuvieron lugar en la UN, fundamentalmente ligadas con el Bienestar Universitario. Como parte de estas, la lucha por las residencias universitarias fue la que mayores implicaciones tuvo en torno a la vida universitaria y, desde septiembre de 1982, se convirtió en un tema de debate público en la ciudad.

Con motivo del crimen contra León, una semana después el Movimiento Estudiantil convocó a un acto de repudio, el miércoles 16 de mayo. La Publicación 16 de mayo ${ }^{1}$ (1984), que circuló en julio de 1984, señala que el evento se desarrolló a partir de diferentes registros e intenciones que marcan la diversidad de iniciativas que caracterizan un movimiento tan heterogéneo como el estudiantil. El repudio se expresó a través de pancartas, poemas, música, y, en el transcurso de la jornada, derivó en un enfrentamiento con la fuerza pública (p. 4).

Aunque estos enfrentamientos eran parte de los repertorios de protesta del estudiantado, por lo cual, eran relativamente recurrentes, los niveles de violencia de esta confrontación fueron mucho mayores que los habituales. Según la información de publicaciones estudiantiles y algunos registros de prensa, la acción estatal incluyó la coordinación de la Fuerza Disponible, el Escuadrón de Motorizados, los servicios de inteligencia del F-2 y el Grupo de Operaciones Especiales de la Policía (GoEs), que ingresaron a la UN haciendo uso de sus armas contra quienes se encontraban adentro; así mismo, los agentes denunciaron haber sido atacados con armas de fuego, explosivos y piedras. Rápidamente, el despliegue de los uniformados venció la resistencia estudiantil y ocupó el campus universitario: hirieron con golpes y disparos a estudiantes, capturaron alrededor de setenta, y causaron destrozos en las residencias

1 Esta es la primera publicación estudiantil que circuló abordando lo acontecido y, en gran medida, estableció los marcos de sentido de estas memorias. En adelante, para evitar confusiones con la fecha, esta publicación se citará como P16M y se hará referencia a ella como Publicación 16 de mayo. 
estudiantiles y los bienes de las personas residentes (P16M, 1984, p. 6; Sandoval y Pinzón, 1992, p. 72).

Figura 1. Residencias femeninas, tras los destrozos causados por la policía.

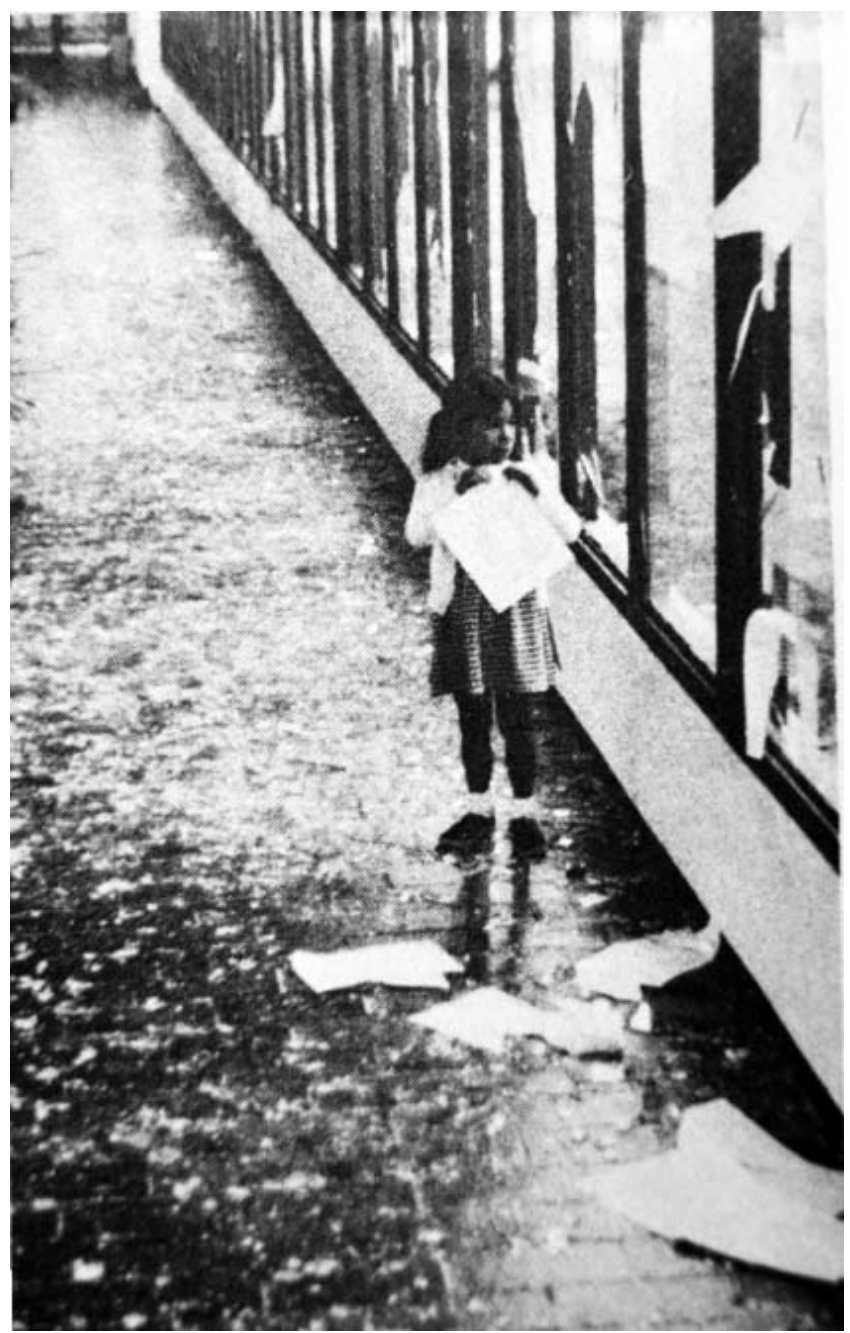

Fuente: revista Cromos, 17 de mayo de 1984. 
El saldo de los acontecimientos violentos entre estudiantes y uniformados ha sido siempre objeto de debates y discrepancias. Del lado de la policía, circuló en la prensa ${ }^{2}$ información sobre 22 agentes heridos, tres de ellos tratados en cuidados intensivos, quienes se habrían recuperado en los días siguientes (Semana, 19 de mayo de 1984). En todos los medios consultados el jueves 17 de mayo, circuló una lista con los nombres de los uniformados lesionados y el tipo de afectación que sufrieron (El Espectador, 17 de mayo de 1984; El Espacio, 17 de mayo de 1984; El Bogotano, 17 de mayo de 1984; El Tiempo, 19 de mayo de 1984).

Por otro lado, las consecuencias que sufrieron los estudiantes han sido históricamente invisibilizadas. La revista Semana, en un artículo crudamente titulado «Plomo, muerte y cierre», señaló que hubo «46 estudiantes heridos y 70 capturados, entre ellos, ocho mujeres» (Semana, 19 de mayo de 1984). Además, la misma fuente destaca el testimonio del sacerdote y profesor de Sociología Jaime Rodríguez, quien junto a «más de cincuenta testigos presenciales (...) aseguraron haber visto cuando a uno de los estudiantes muertos en el enfrentamiento se le colocaba una capucha con el distintivo del M-19 que, según estas mismas versiones, la propia Policía portaba» (Semana, 19 de mayo de 1984) (el énfasis es mío).

Los estudiantes, por su parte, denunciaron casos de desapariciones y asesinatos masivos y, con el tiempo, nombraron lo ocurrido como la masacre del 16 de mayo. Sobre las magnitudes del daño, sin embargo, no existe un consenso y, cuando se presentan cifras, estas tienden a variar según la fuente: en los testimonios presentados en la Publicación 16 de mayo y en la información publicada por el periódico El Bogotano (18 de mayo de 1984), se encuentra información de por lo menos seis víctimas. En una tesis de grado de la carrera de Trabajo Social de la

2 En esta investigación se tuvieron en cuenta medios de comunicación de circulación masiva como El Bogotano, Revista Cromos, El Espectador, El Espacio, El Tiempo y la revista Semana. Agradezco a Sergio Hurtado por compartir una gran parte de estos materiales, al igual que por la actitud solidaria que ha tenido para discutir colectivamente este tipo de temas. 
UN, Sandoval y Pinzón (1992) afirman que el saldo fue de «22 policías heridos y datos no confirmados hasta el presente de siete estudiantes desaparecidos» (p. 72). En un comunicado firmado por la Asamblea Estudiantil de la UN (1988), circulado con motivo de la conmemoración de los hechos en 1988, se señala que «[el 16 de mayo] la fuerza pública invadió los predios de la Universidad, masacrando a más de 20 compañeros y desapareciendo a otros tantos». Mientras que Aponte (2006), en su trabajo de grado de la carrera de Ciencia Política de la UN, indica que «fueron asesinados varios estudiantes. El número exacto no se conoce, pero existen versiones que hablan de diecisiete. También fueron desaparecidos algunos más» (p. 26).

De hecho, el significante indeterminado es uno de los más estables y recurrentes en los documentos producidos por el Movimiento Estudiantil, cuando se refiere al número víctimas. Asimismo, hasta ahora, sus nombres no han podido ser establecidos con seguridad, pese a que, como se verá en los documentos del propio movimiento y en la prensa, hay información particularizada de posibles víctimas de los acontecimientos violentos.

Este carácter abierto ha sido usado por posiciones que niegan las denuncias de los estudiantes o consideran que lo que pasó es de proporciones mucho menores a las denunciadas. Desde estas posiciones, se cuestionan las memorias estudiantiles y se pregunta por las pruebas, los números específicos, los nombres completos y las denuncias en las instituciones. La falta de respuestas definitivas a estos cuestionamientos define en gran medida los límites de la narrativa estudiantil, es decir, de lo que por ahora ha podido ser contado sobre lo ocurrido. Destaco por ahora, en tanto que la puesta en marcha del Sistema Integral de Verdad, Justicia, Reparación y No Repetición (SIVJRNR) y, específicamente, de la Comisión para el Esclarecimiento de la Verdad (CEV), además del convenio adelantado en la primera mitad del 2019 entre la CEV y la UN para investigar el lugar de esta en el conflicto, constituyen una ventana de oportunidad para ampliar los marcos de referencia sobre lo que se 
conoce acerca de situaciones violentas como esta y garantizar plenamente el derecho de las víctimas y la sociedad a la verdad.

En razón a lo anterior, y dada la experiencia que he tenido como activista estudiantil y como investigador sobre este tema, puedo asegurar que, para las personas afectadas de diferentes maneras por los eventos del 16 de mayo de 1984, sus memorias están revestidas de realidad, cargadas de dolor y carentes de justicia. Esto ha configurado de manera silenciosa subjetividades a partir de las cuales las personas se reconocen como víctimas o sobrevivientes del 16 de mayo y, en muchos casos, esto se ha sobrellevado con silencios dolorosos de más de tres décadas.

Sin embargo, las versiones que niegan o desestiman las denuncias han planteado una disyuntiva entre la experiencia de quienes se reclaman como víctimas o sobrevivientes y las posibilidades de "probar» su afectación. Esta disyuntiva se inscribe de forma problemática en la relación entre las memorias de las víctimas y la construcción de verdades históricas. En estos casos, opera generalmente una profunda desconfianza hacia el testimonio y las personas testimoniantes, sobre lo que se ha sustentado en gran medida la narrativa estudiantil. Más aún, la condición de subalternidad que define a memorias como estas, hace que las pruebas disponibles en registros distintos a los testimonios sean, en muchos casos, igualmente silenciadas y minorizadas.

Como consecuencia de lo expresado y de su acción en la construcción social de memoria y olvido, pese a las iniciativas de conmemoración que se despliegan anualmente desde 1985 y a la presentación de testimonios de víctimas o sobrevivientes de los acontecimientos en estos espacios, no existe un consenso (ni siquiera entre el estudiantado) sobre considerar o no este como un caso de victimización contra las y los universitarios.

Incluso, la información disponible en registros de prensa y en documentos producidos por el Movimiento Estudiantil — de gran relevancia 
para comprender lo sucedido - ha sido usualmente desestimada tanto por las posiciones negacionistas como por las investigaciones académicas existentes sobre el tema.

Esta cuestión será abordada en el presente artículo, a partir de la pregunta: ¿sobre qué elementos se sustentan las versiones que dieron lugar a que este fuera denunciado como un crimen contra el estudiantado?

Con este objetivo, se indagarán fuentes surgidas en los días y meses siguientes a los acontecimientos de mayo de 1984, principalmente, la prensa de la época y materiales producidos por el Movimiento Estudiantil, especialmente, la Publicación 16 de mayo ${ }^{3}$. Esta revisión posibilitará el reconocimiento de las posturas negacionistas — con un fuerte arraigo social, pero con escaso registro documental-y de las graves denuncias que, aun cuando pueden ser sustentadas con fuentes documentales y testimoniales, han sido poco visibles en los escenarios en los que se celebra este tipo de discusiones.

\section{Contexto: casa tomada}

Durante 1982 y 1984, el núcleo articulador de los conflictos universitarios en la UN-Sede Bogotá se estableció alrededor de la continuidad o el cierre del programa de Residencias Estudiantiles. Desde noviembre de 1976, a partir de la resolución 185, el Consejo Superior Universitario (CSU) había ordenado «suspender indefinidamente, a partir de la fecha, el programa de Residencias Estudiantiles, adscrito a la Dirección de Estudiantes» (CSU, 1976). Sandoval ${ }^{4}$ y Pinzón (1992) cuentan que este fue el segundo intento de cierre tras un primer periodo de clausura

3 La mayoría de las fuentes de archivo consultadas para este artículo han sido tomadas del fondo del proyecto Archivos del Búho.

4 Beatriz Sandoval era militante anarquista, estudiante de último semestre de la carrera de Trabajo Social y coautora de una tesis que tiene como tema el Movimiento Estudiantil. Murió en medio de una confrontación con la policía en 1991, durante la conmemoración de los acontecimientos violentos del 16 de mayo. 
que duró ocho meses en 1972. Ante la resistencia estudiantil, el desalojo se llevó a cabo con tutela del ejército: «eran las épocas en que los cercos a "Gorgona"5 duraban semanas, y eran violentos los enfrentamientos entre la tropa y los estudiantes que se atrincheraban dentro» (Semana, $1 .^{\circ}$ de noviembre de 1982).

Aunque, en ambos casos, el principal argumento para el cierre era que el Gobierno y la institución veían a las residencias como focos subversivos, las declaraciones ofrecidas en 1982 a la Revista Semana por el ingeniero Contreras, director de Bienestar Estudiantil, indican que, en realidad, convergía una serie de factores: «aunque, a partir del 76, cuando el Gobierno cerró las residencias aduciendo razones de orden público, se han venido realizando estudios de factibilidad de apertura, y ha sido el factor económico el determinante para no reabrirlas» (Semana, $1 .^{\circ}$ de noviembre de 1982).

El cierre de los servicios de alojamiento, que podían albergar aproximadamente 4000 residentes $^{6}$, resultó efectivo, manteniendo en funcionamiento poco más del $10 \%$ de su capacidad. Los edificios de residencias Santander y Antonio Nariño ${ }^{7}$ fueron cerrados, así como la mayoría de los espacios de los edificios Uriel Gutiérrez y la Unidad Camilo Torres. Las residencias femeninas (hoy edificio Manuel Ancízar) siguieron funcionando

5 De esta manera se conocía el edificio de residencias Uriel Gutiérrez. La referencia es a la isla Gorgona del pacífico colombiano, usada como cárcel por los gobiernos del Frente Nacional desde 1960. La relación, seguramente, tiene que ver con su ubicación alejada del resto del campus, cuyos alrededores se inundaban en los días de lluvia, según cuentan.

6 En el Boletín de Estadística de la UN solamente hay información de los espacios ocupados regularmente, es decir, de las residencias femeninas, 10 de mayo y algunos bloques de casados. Sin embargo, la cifra total de 4000 residentes fue manejada por las directivas universitarias, frecuentemente, en fuentes como la prensa. Un ejemplo puede encontrarse en el artículo "Día de decisiones ante posible cierre», publicado en El Bogotano el 28 de marzo de 1984.

7 Tras la toma de las residencias en 1982, este edificio fue renombrado como residencias Alberto Alava, en memoria del profesor de Derecho de la UN, militante revolucionario y defensor de presos políticos, asesinado el 20 de agosto de 1982 . 
y recibieron a la mayoría de las residentes regulares durante estos años (353 para en el año 1983); además, se mantuvo una parte de las residencias 10 de mayo, donde vivían 126 estudiantes y siete profesores, y algunos espacios dedicados a parejas casadas donde residían 55 personas. En total, serían 534 residentes los que se mantendrían habitando estos espacios en los años que siguieron al cierre (UN, 1983, p. 28).

Durante el año 1982, tuvieron lugar varios acontecimientos decisivos en esta historia. Por un lado, entre el 4 de marzo y el 13 de septiembre, se adelantó un proceso de desaparición sistemática de activistas estudiantiles que tuvo características similares a las tácticas usadas por las dictaduras militares del Cono Sur. Estos crímenes se dieron a conocer como el Caso colectivo 82 por la acción de los familiares de los estudiantes agrupados en Asfaddes $^{8}$. En este caso, a partir de la acción conjunta entre los organismos de inteligencia del F-2 y el grupo Muerte a Secuestradores (MAS), una docena de personas fueron desaparecidas, en su mayoría estudiantes de la UN y la Universidad Distrital ${ }^{9}$. Como parte de este contexto represivo, el 20 de agosto de 1982 fue asesinado el profesor de Derecho de la UN, militante revolucionario y defensor de prisioneros políticos, Alberto Alava. Estos hechos motivaron una movilización universitaria en repudio a la represión, que resultó siendo catalizadora de otros reclamos: « [abrió] las posibilidades al movimiento de recuperación de residencias que se materializó en la toma de estas el 21 de septiembre del 82» (FER-Sinpermiso ${ }^{10}, 1988$, p. 5).

Tras la toma, la administración universitaria, en cabeza del rector Fernando Sánchez Torres, optó por una posición que se movía entre el diálogo y la intervención; aunque es un hecho que priorizó lo primero. Resultado de lo anterior es el Acuerdo 32 del 11 de abril de 1984, en el

8 Asociación de Familiares de Detenidos Desaparecidos.

9 Para ampliar este tema, se puede consultar el proyecto Vidas Silenciadas: www. vidassilenciadas.com/caso-colectivo-82/

10 Frente Estudiantil Revolucionario-Sinpermiso: organización estudiantil. 
que, luego de meses de negociación entre las directivas y los representantes estudiantiles, se definieron las características de la regularización de las residencias y sus organismos de administración, supervisión y control. Para hacer efectivo este proceso, las residencias fueron desalojadas voluntariamente por los ocupantes el mismo día que se firmó el Acuerdo 32. En el año 2003, Sánchez Torres señaló en UN Periódico (2003) que «los altos mandos militares eran partidarios de tomarlas a la fuerza, para lo cual tenían previsto un plan que no llevaría más de cinco minutos». Ante la posibilidad de que esto decantará en muertos y heridos el entonces rector siempre rechazó esta posibilidad, por lo que consideró el Acuerdo 32 y la regularización que iniciaba el 11 de abril como «un triunfo del diálogo y la concertación».

Pese a esto, la situación producida por la toma de las residencias estableció un nudo complejo con hilos y actores diversos. Por las características de la conflictividad social del país, esta disputa había dejado de ser una cuestión estrictamente universitaria, lo que se evidencia en el desfase evidente entre los acuerdos de abril, los planes de la toma militar y los acontecimientos violentos que estarían por venir en mayo.

En las residencias intervenían, en primer lugar, los estudiantes que las ocupaban, las organizaciones estudiantiles, sus representantes y las directivas universitarias. Sin embargo, es un hecho que también hacían presencia e influían en la situación algunas expresiones estudiantiles de las organizaciones armadas, grupos narcotraficantes, organismos de inteligencia encubiertos y la acción franca de la Policía, que cada tanto hostigaba a los residentes. Respecto a este último punto, Luis Higuera (2018), representante estudiantil en la negociación con la administración universitaria, señaló en entrevista: «El Estado no deja de hacer lo suyo. De manera permanente, en las horas de la noche, se toman las residencias para intentar desalojos no autorizados. Lo que resultaba en enfrentamientos nocturnos con la Policía y con el Ejército». 
La crónica Ciudad Blanca, Ciudad Fuego, de Juan Sebastián Salamanca (2007), ilustra a partir de la ficción la compleja diversidad de actores que intervenían en la situación, a partir de una conversación entre dos amigos basada en testimonios:

Su rostro jovial se pone serio y su jerga popular se transforma en expresiones de todo un intelectual. Que las residencias se tomaron porque se tenía que dar solución a un problema vital para la comunidad, que hubo encargados por cada pasillo, por cada piso y por edificio, que se debían rotar cada cierto tiempo, pero que con los meses, los jíbaros - algunos del Cartel de Bogotá- aprovecharon para penetrar en esas estructuras y alcanzaron a tener control sobre algunos pisos y chocaron con el M-19, el ELN y otros grupos y con una gran masa de estudiantes que rechazaban a esta gente que trajo el bazuco a la Universidad y que incluso, ahí mismo, dentro de los edificios, fabricaban el perico y pauperizaban más y más a los pelaos, cada vez más esclavos de esa nueva droga. (p. 8)

Las violencias desplegadas en este contexto son, entonces, de un carácter muy complejo. Además del caso de Chucho León, ocurrieron otras dos muertes relacionadas con esta situación. Hacia finales de 1983, el estudiante de veterinaria Julio Barrera murió al caer del quinto piso del edificio Uriel Gutiérrez, al parecer cuando intentaba escapar por una ventana del enfrentamiento entre expendedores de droga y una organización denominada Muerte a Pepos. El segundo caso se presenta el 14 de mayo en el sur de Bogotá con el asesinato del médico Luis Armando Muñoz González, director académico de la carrera de Medicina, quien habría denunciado la presencia de expendedores en las residencias y habría impedido su retorno en el proceso de regularización (UN Periódico, 2003).

Tras los acontecimientos violentos del 16 de mayo, el rector Fernando Sánchez Torres renunció y el Consejo Superior Universitario ordenó un cierre de la UN que se extendió por un año. Durante este periodo, en octubre de 1984, el CSU (1984) ordenó la clausura definitiva de los 
programas de residencias estudiantiles y del servicio de alimentación que proporcionaba la universidad, para, finalmente, con el Acuerdo 100 de diciembre de 1984, definir que «los préstamos-beca reemplazan los sistemas de Cafetería y Residencias Universitarias que existían en la Sede Bogotá».

Con estos cambios, la universidad retomó funciones en abril de 1985 . No obstante, el total de personas matriculadas tras el periodo de cierre se vio sensiblemente reducido. A partir de la información presentada en el libro Estadísticas e indicadores de la Universidad Nacional 1970-1995 (1996), se puede deducir que, entre 1974 y 1984, la UN Sede Bogotá tuvo en promedio 16699 estudiantes. Esta misma fuente registra un total de 16537 para el primer semestre de 1984. Dato que presenta un cambio significativo en la reapertura de abril de 1985, con la matrícula de 1262 estudiantes menos que el año anterior, llegando a un total 15268 y la significativa reducción de un $8 \%$ de los matriculados. Si bien en los años siguientes esta cifra aumenta progresivamente, solo sería hasta 1990 cuando la Universidad alcanza el número de personas matriculadas registrado en 1984.

En un documento publicado por el Movimiento Estudiantil, presentado durante la reapertura de la UN en abril de 1985, se señala que, como consecuencia de las nuevas políticas del Bienestar Universitario, «los estudiantes de provincia y de menores recursos se han visto forzados a abandonar la U, con la consiguiente elitización y autofinanciación que traslucen los préstamos “condonables” o créditos estudiantiles» (CGTUN $\left.{ }^{11}, 1985\right)$. Aunque en las fuentes estadísticas de la UN que han sido consultadas no existen reportes socioeconómicos que permitan confirmar o desmentir parte de lo denunciado por los estudiantes, la información existente sobre el lugar de procedencia les otorga la razón: para 1984, la población de estudiantes bogotanos constituía un $54 \%$ del total, dato que, sumado a la población de estudiantes de

11 Coordinadora de Grupos de Trabajo de la UN. 
Cundinamarca, llegaba a representar un $65 \%$. Las fuentes muestran que en los años siguientes a 1984 esta cifra fue en franco aumento y llegó a representar un 82,5 \% de la población de la UN en 1995 (UN, 1985; UN, 1996).

\section{El negacionismo}

Ante las denuncias del estudiantado tras los acontecimientos violentos del miércoles 16 de mayo de 1984, emergieron versiones paralelas que las negaban y confrontaban. Una de estas primeras acciones fue adelantada por el comandante del Departamento de Policía de Bogotá, el general José Luis Vargas Villegas, quien «negó las versiones de varios testigos que aseguran haber visto ingresar a los predios de la Ciudad Universitaria a varios grupos de la escuadra motorizada de la Policía» (El Espectador, 17 de mayo de 1984). En la misma línea, para la revista Cromos (17 de mayo de 1984), Vargas Villegas señaló: «Estoy seguro que algunos periodistas, si son serios, estarían encantados de poder tomar fotografías de los policías destruyendo las residencias. Yo quiero ver los muertos. Quiero los nombres de los desaparecidos». No obstante, tanto la entrada de los policías, como las afectaciones que estos mismos provocaron sobre las residencias y las personas que allí se encontraban fueron hechos registrados en fotografías y testimonios circulados por el Movimiento Estudiantil y medios masivos de comunicación.

Días más tarde, en un artículo que circuló en el periódico El Tiempo (19 de mayo de 1984), las denuncias fueron desestimadas al expresar que, «al preguntarles [a estudiantes y profesores] dónde estaban los muertos, cuáles eran los nombres de los presuntos desaparecidos, se quedaban mudos. Han pasado más de 72 horas (...) y aún no aparece el primer muerto». Este artículo fue firmado por Ayatollah, seudónimo usado por Rafael Santos Calderón, quien, además, señaló de modo justificatorio que: 
Figura 2. Policía motorizada en los predios universitarios.

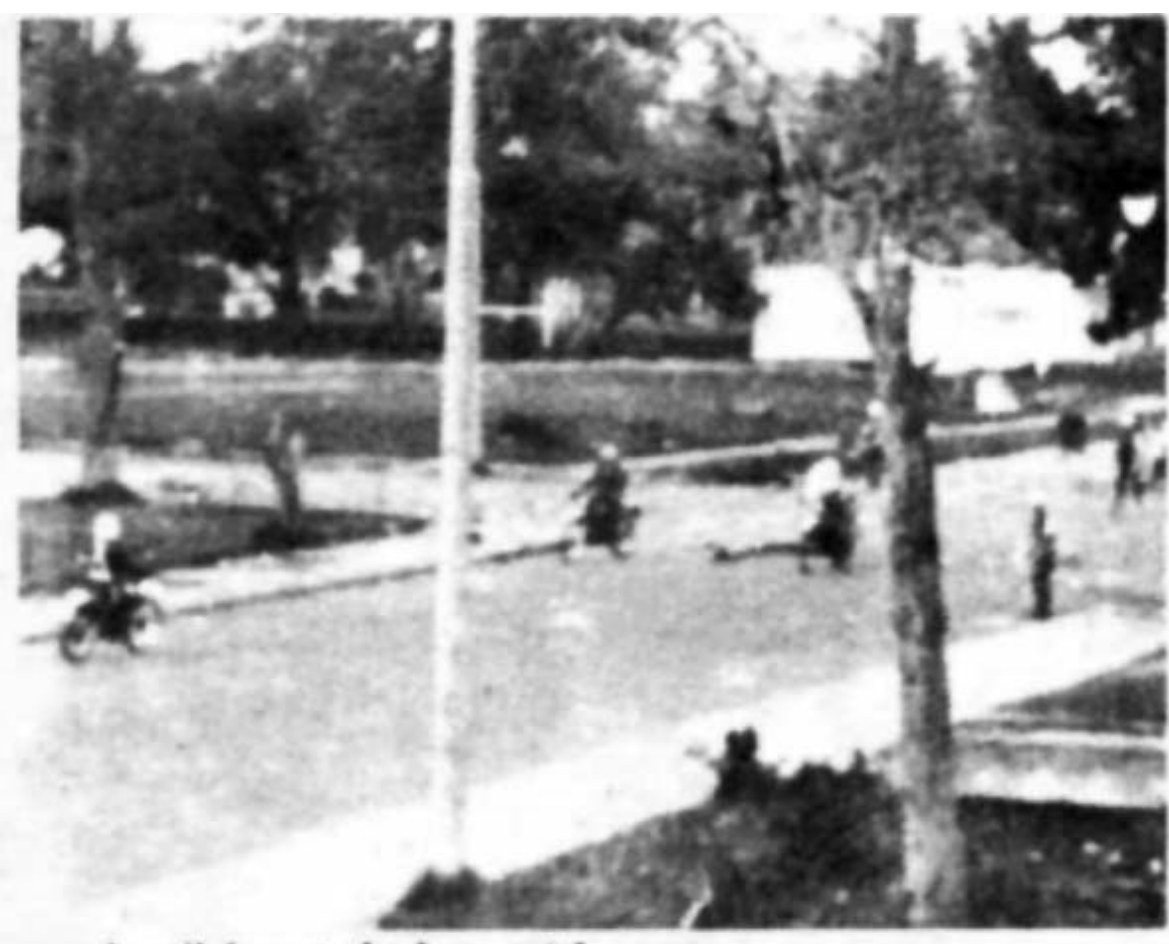

... y lo polieis motorizada no antib.

Fuente: P16M, 1984.

En las instalaciones universitarias se han enquistado como un tumor maligno esa mafia de agitadores, violentos y amorales, que no conocen el límite de la ley y que están dispuestos a todo, inclusive asesinar, con el único ánimo de hacer desorden instalar el caos, sin propósitos claros de lo que persiguen distinto a exacerbar los ánimos y precipitar el cierre de la Universidad.

Años más tarde, en mayo de 2003, en un momento cercano al aniversario número 20 de los acontecimientos y al inicio de la segunda administración de Marco Palacios (rector que reemplazó a Sánchez 
Torres y lideró las principales reformas desarrolladas desde 1984), el exrector Sánchez Torres se sumó públicamente a este tipo de posiciones con un relato detallado en el que presenta su versión de los acontecimientos. Lo hizo a partir del artículo titulado «En honor a la verdad», publicado en UN Periódico (2003), en el que plantea:

El 16 de mayo, apenas transcurridos dos días del asesinato del doctor Muñoz, tuvo lugar en predios de la Universidad un feroz enfrentamiento entre la Policía y sujetos embozados, con un saldo de 22 heridos, a bala unos, con ácidos y granadas otros. Hubo 123 detenidos, la mayoría de ellos encapuchados (...).

Al recordar públicamente estos ingratos acontecimientos, solo pretendo desvirtuar las visiones que se dan por ciertas y que, por lo mismo, pueden incidir desfavorablemente en la marcha de la Universidad. (el énfasis es mío)

Aunque Sánchez no especifica si los heridos fueron policías o estudiantes, parece claro que se refiere a los 22 policías heridos que la prensa documentó en detalle. Así mismo, no solo evita hacer referencia alguna a la existencia de denuncias de los estudiantes, sino que presenta de manera abierta la intención del artículo por desvirtuar las visiones que se dan por ciertas, con lo que se inscribe explícitamente en la disputa por los relatos que dan cuenta de este pasado.

Vale la pena señalar que, de manera inversa, los documentos del Movimientos Estudiantil y las investigaciones existentes que hacen referencia a lo ocurrido el 16 de mayo de 1984 están centrados en la violencia de la Policía sobre los estudiantes. La tesis de Sandoval y Pinzón (1992), citada anteriormente, parece ser el único documento en el cual se presenta información sobre policías heridos ${ }^{12}$. Sin embargo, en ninguno de los materiales de archivo del Movimiento Estudiantil que han sido

12 Las autoras plantean que el saldo de los acontecimientos fue de «22 policías heridos y datos no confirmados hasta el presente de siete estudiantes desaparecidos» (Sandoval y Pinzón, 1992, p. 72). 
consultados se tiene en cuenta a los 22 policías heridos que detalla la prensa. Ambos son, en definitiva, silencios constitutivos de cada uno de estos relatos.

La comprensión de estos silencios debe considerar el carácter subalterno de las memorias estudiantiles y los alcances que cada relato tiene en relación con la construcción de legitimidad y poder. Muestra de eso es el imaginario dominante que entiende que si las y los estudiantes son usuarios de la violencia esto «justifica» lo cometido en su contra, o que de los policías se puede obtener información que detalla caso por caso sus nombres e, incluso, sus edades y las heridas que sufrieron.

Ante la falta de respuestas completas y aceptadas a los cuestionamientos hechos a las denuncias estudiantiles, y como consecuencia de la acción de quienes como Sánchez Torres y Santos Calderón han intervenido públicamente en su negación, versiones como las que han sido presentadas antes se han instalado como dominantes en torno a este caso. Se pueden reconocer en discursos públicos como los citados, aunque su registro documental es verdaderamente escaso en comparación con su arraigo social.

Aun cuando algunos sectores del estudiantado adelantan anualmente iniciativas de memoria, hoy en día no se puede afirmar que dentro del propio Movimiento Estudiantil haya una aceptación general de las denuncias de los estudiantes de los años ochenta; de hecho, considero que en algunos sectores se ha instalado una duda sobre la veracidad de las mismas. Esto no tiene hasta ahora soportes documentales y ninguna organización o vocería ha sostenido públicamente una posición contraria a las denuncias. Sin embargo, al conversar sobre el tema con algunos líderes de diferentes organizaciones, encontré que en ciertos casos estos reconocían las memorias del 16 de mayo como una especie de mito y se referían al suceso como algo no necesariamente verdadero. 


\section{Las denuncias}

Portelli (1989) asegura que «la historia (...) es una invención a la que la realidad aporta los propios materiales. No obstante, no es una invención arbitraria» (p. 6) ¿De qué materiales entonces está compuesto el relato que denuncia casos múltiples de victimización en los acontecimientos del 16 de mayo? Además de los testimonios de víctimas o sobrevivientes y demás testigos de los acontecimientos - que en gran medida han soportado la transmisión de estas memorias-, en lo que resta de este artículo se presentarán algunos elementos que pretenden servir como un aporte a la comprensión de lo sucedido.

\section{El caso de William Mayorga y los testimonios de la Publicación 16 de mayo}

La Publicación 16 de mayo fue un documento circulado en julio de 1984, que sintetiza el esfuerzo de sus productores por recoger información disponible sobre lo ocurrido el 16 de mayo del mismo año en la UN. Sus productores fueron un grupo de estudiantes vinculados a proyectos comunicativos como Corriente Alterna de la Facultad de Ingeniería, que sustentaron su relato en fuentes de prensa y en el testimonio de testigos ${ }^{13}$.

El documento inicia con la presentación de un informe sobre el crimen cometido contra Chucho León, que incluye información sobre su asesinato y el dictamen de medicina legal de Pasto, donde se da cuenta de las torturas que sufrió. Los productores resaltan la importancia de este último, dado que, anteriormente, había circulado un primer dictamen forense practicado en Cali, en el que las torturas fueron obviadas y el profesional médico solo reseñó las heridas mortales (p. 3).

13 Entre el grupo que realizó entrevistas se encontraba el estudiante de ingeniería Orlando Quintero Páez, quien posteriormente alcanzaría importante visibilidad por el proceso de ruptura procesal con el sistema de justicia que adelantó siendo preso político y militante del Ejército de Liberación Nacional. 
A partir de testimonios y fotografías, la Publicación 16 de mayo buscó probar lo que las autoridades y algunos periodistas habían negado abiertamente en medios de comunicación: la presencia de miembros de los servicios de inteligencia y motorizados en los acontecimientos, las afectaciones a las víctimas de disparos efectuados por la policía y las situaciones que se denuncian como casos de desaparición forzada.

Pese a que se ha dicho que en estas memorias no han podido asegurarse los nombres de las víctimas, en la portada de este documento se presenta el caso de un estudiante con nombre y apellido: William Mayorga. Al respecto la publicación señala:

(...) después de más de un mes de recibir casi día a día informes sobre posibles desaparecidos, es de nuestro conocimiento el asesinato del compañero de Antropología William Mayorga, quien se encontraba realizando la monografía requerida para obtener su grado como antropólogo. Su cadáver fue encontrado en el barrio Santa Rita el 19 de junio - presentaba dos impactos de bala en su cabeza y golpes en diferentes partes del cuerpo- y fue entregado a su familia con la orden perentoria de ser enterrado inmediatamente. Su sepelio fue el 20 de junio. (p. 1) (el énfasis es mío)

Este caso plantea uno de los retos más complejos para entender las memorias del 16 de mayo, pues esta publicación es el único documento que menciona lo sucedido con William Mayorga: ipor qué si uno de los principales argumentos de los detractores de las denuncias estudiantiles era la ausencia de nombres de las víctimas, este caso resultó ocultándose en el devenir de estas memorias? Aunque esta pregunta excede por mucho los límites e intereses de este artículo, considero pertinente explorar una perspectiva que pueda ser profundizada o rebatida en otros trabajos: ante la imposibilidad de comprobar plenamente otros casos de víctimas estudiantes como los que eran recibidos casi día a día por los productores de este documento - e incorporar así sus nombres e historias al relato-, el anonimato terminó funcionalizandose en la narrativa, para sostener el carácter masivo de las afrentas tanto 
en relación con las denuncias de personas heridas, detenidas, asesinadas y desaparecidas, como frente a las consecuencias sociales que acarrearon las reformas al sistema de Bienestar Estudiantil referidas anteriormente. En otras palabras, es posible que, pese a la certeza de hechos como el caso de Mayorga, en el devenir de estas memorias, resultó más útil la denuncia de un crimen masivo pero anónimo que la reivindicación de un solo caso.

Por otro lado, cuando los productores aseguran haber recibido casi día a día información sobre posibles desaparecidos, dan cuenta de un contexto de producción marcado por la incertidumbre, sobre el cual no pudieron tener mayores certezas. De haber sido así, la información que hubieran presentado sobre posibles personas desaparecidas habría sido seguramente mayor; no obstante, la posibilidad de otros casos ronda la afirmación como un fantasma y, como se verá, circuló en otros medios como la prensa masiva.

Además de este caso, a partir de los testimonios sobre el enfrentamiento entre estudiantes y policías, el documento presenta seis casos distintos de estudiantes heridos, algunos de estos presuntamente desaparecidos. Los testimonios son tomados de la palabra de nueve personas -la mayoría de ellas identificada con nombre y número de identificación-, citadas por partes, de tal modo que, entre todos construyen una única narrativa. Es decir, no son presentados uno por uno, sino como fragmentos organizados en función de una sola secuencia definida de la siguiente manera: los policías disparan contra los estudiantes, entran a las residencias, atrapan gente que intentaba esconderse, destruyen a su paso edificios y bienes y capturan personas que intentaban escapar en los alrededores de la universidad. Estos son algunos de los testimonios:

Un muchacho que era perseguido por la Policía fue alcanzado por esta y golpeado brutalmente, después de lo cual uno del grupo policial vestido de civil le disparó dos veces. El cuerpo fue arrastrado y llevado con destino desconocido (...). 
[El policía] lo agarró por el hombro con la mano izquierda y le disparó en el hombro izquierdo por la espalda. Toda la gente gritó, yo no me podía mover, caí de rodillas; uno de los vestidos de civil le levantó la camisa al muchacho y el muchacho botaba mucha sangre. Cuando cayó, yo no sé si cayó vivo o muerto o si se desmayó. Después, unos policías le pusieron algo blanco en la cara, lo taparon y ya no lo vi más (...).

Corrían dos estudiantes en medio del pánico general que cundía en la universidad. Ellos buscaban un sitio de protección y detrás de ellos un piquete de uniformados y tres civiles quienes dispararon a quemarropa a uno de los estudiantes que huía afanosamente. El acompañante de este, al parecer otro estudiante, al ver semejante escena tan escabrosa se detuvo con las manos en alto y fue aprehendido de inmediato por los mismos policías y golpeado brutalmente. Luego, lo obligaron a cargar el cuerpo de su compañero abaleado y lo guiaron hacia la jaula a punta de bolillo. Es importante anotar que, la policía, al levantar el cuerpo del abaleado, le colocó en el rostro una capucha del M-19. Esto fue presenciado aproximadamente por unas 50 personas que a esa hora se encontraban escondidas en el edificio de Sociología. (P16M, 1984, pp. 5 y 14)

En la información presentada en los testimonios hay dos elementos constitutivos del relato que vale la pena destacar: la sugerencia de un posible caso de desaparición, cuando los policías se llevan un cuerpo con destino desconocido, y la presencia de los policías de civil, en quienes se fija la acción de disparar.

Finalmente, el documento reproduce fotografías y una radiografía de algunos de los heridos, las cuales fueron publicadas en la prensa de circulación masiva. En una de estas, un estudiante presenta una herida de bala en el estómago (El Bogotano, 17 de mayo de 1984) y otro que recibió dos disparos en su rostro (Cromos, 17 de mayo de 1984); en relación a este caso, la radiografía publicada exhibe el orificio de salida de una bala en el pómulo derecho de la víctima: 
Figura 3. Radiografía del estudiante herido de bala en el rostro ().

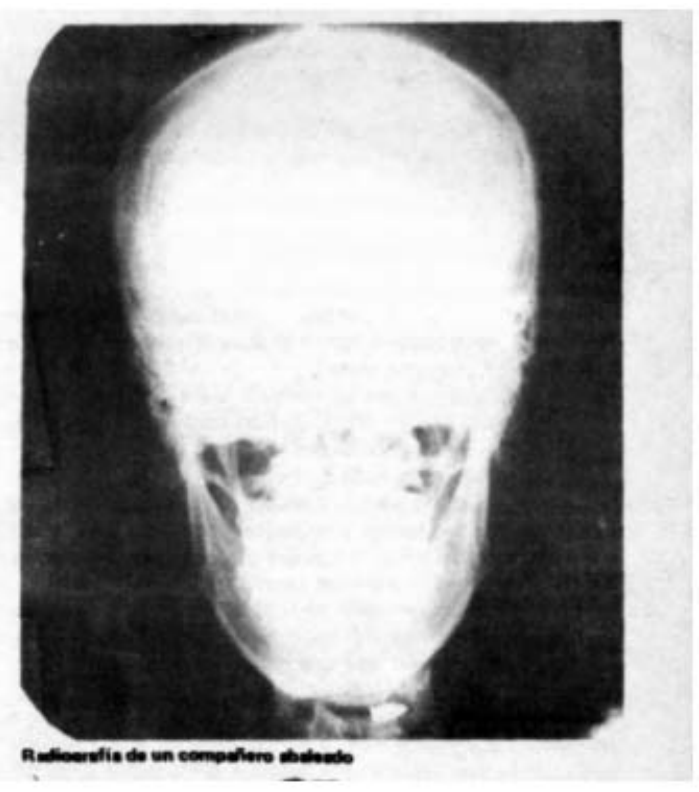

Fuente: P16M, 1984.

\section{Registro en prensa: «Hay estudiantes muertos»}

Esta radiografía fue reproducida por los estudiantes tras su publicación en la revista Cromos (17 de mayo de 1984), medio en el que circuló junto a información sobre las heridas de bala que el estudiante recibió en su rostro y los golpes y heridas que le fueron causados en el resto del cuerpo. La publicación incluye, además, una fotografía del mismo estudiante con una mano enyesada, a raíz de los golpes, mientras que con la otra cubre su rostro. Esta fuente pudo contactar al herido, quien aseguró llamarse Andrés y tener 19 años; así mismo, compartió detalles de su caso: "pasaba por Odontología hacia la calle 26. Eran las 3 y media de la tarde. Escuché que disparaban. Perdí el equilibrio y sentí calor». 
Este tipo de información circuló masivamente, con lo cual, las denuncias sobre estas afectaciones no fueron exclusivas de los y las estudiantes; de hecho, estas publicaciones derivaron en la suma de algunos medios a la discusión pública sobre lo acontecido. El Bogotano (17 de mayo de 1984, por ejemplo, interpeló al general Vargas Villegas, al señalar que, aunque "[este] desmiente la versión de muertos (...) en esta edición les presentamos fotos exclusivas de uno de los encapuchados muertos». Esta afirmación alude a una de las fotografías en las que se registró el momento del ingreso de la Policía y el impacto de bala que recibió en ese momento uno de los manifestantes, imagen acompañada de un pie de foto que plantea: «Este encapuchado, herido de bala en el estómago, fue fotografiado cuando era llevado a un hospital desconocido. Murió poco después».

Figura 4. Impacto de bala recibido por uno de los manifestantes.

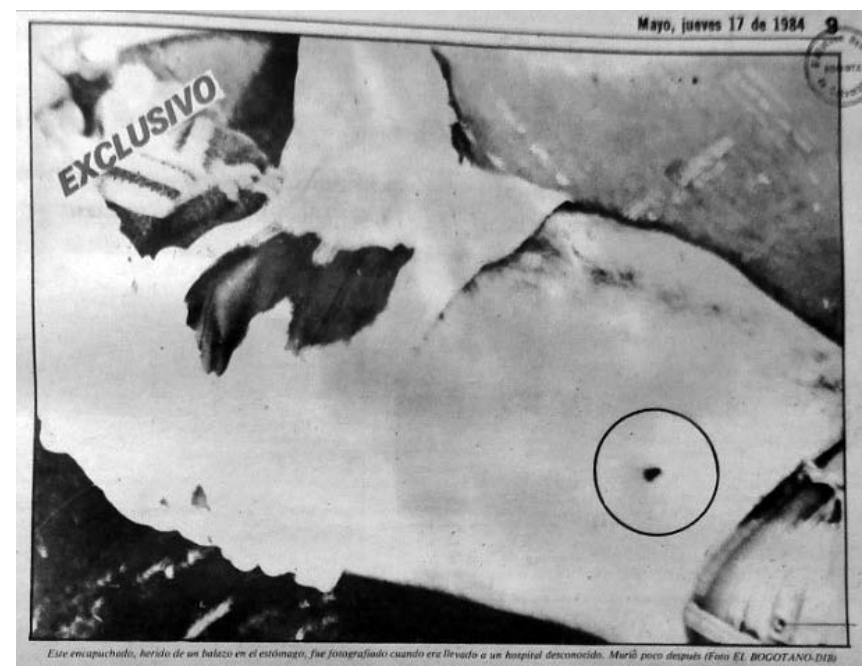

Fuente: El Bogotano, 17 de mayo de 1984.

Esta fuente destacó también que un centenar de estudiantes habrían sido detenidos y que por lo menos habría tres muertos; igualmente, el 18 de mayo publicó un titular que aseguraba: «Hay estudiantes muertos! », 
acompañado de información proporcionada por los estudiantes, a partir de la cual estableció una lista de seis personas desaparecidas: Juan Carlos Lozano, Lisímaco Ovalle, Samuel León, Carlos Julio Reyes, Nubia Cecilia Montoya y Clara Inés Días, lista seguida de la afirmación: «buscamos por lo menos diez cadáveres que se llevó la Policía» (El Bogotano, 18 de mayo de 1984). Cuenta, además, que, pese a haber negado públicamente la posibilidad de que hubiera habido muertos o desaparecidos, en la propia noche del 16 de mayo, el general Vargas Villegas se reunió con el ministro de Defensa y el director nacional de la Policía para analizar la situación «y establecer el paradero de las estudiantes María Cecilia Montoya y María Eugenia Bastidas, quienes fueron dadas por desaparecidas» (El Bogotano, 18 de mayo de 1984). En cuanto a Lisímaco Ovalle y Nubia Cecilia Montoya, sus nombres pudieron encontrarse en las listas de estudiantes detenidos presentadas por la Publicación 16 de mayo; sin embargo, frente al resto de casos señalados por El Bogotano, no ha sido posible encontrar mayor información que confirme o rebata las denuncias de desaparición. Por lo cual considero que este es uno de los elementos más importantes a tener en cuenta en futuras investigaciones al respecto de este tema.

Finalmente, el 19 de mayo, en otro reportaje de El Bogotano (19 de mayo de 1984), se ilustró la crudeza de los acontecimientos a partir de la propia experiencia del exrector Sánchez Torres:

También se conoció que aún se siguen encontrando huellas de sangre dentro de los edificios de las diferentes facultades que, según los estudiantes, corresponden a los alumnos heridos y desaparecidos.

En la facultad de Medicina, el mismo rector pudo constatar las huellas ensangrentadas de un pie, que quedaron como mudos testigos del pánico vivido en los predios universitarios durante la tarde del miércoles. Las huellas son verdaderamente patéticas y reflejan el paso de un herido, por el pasillo, en un solo pie; antes de caer o ser arrastrado. También, en las 
paredes de los salones fueron encontrados plomos y en un vidrio se observa el paso de un proyectil.

\section{Figura 5. A la izquierda, ingresa la Policía al campus; a la derecha, los estudiantes cargan un herido.}

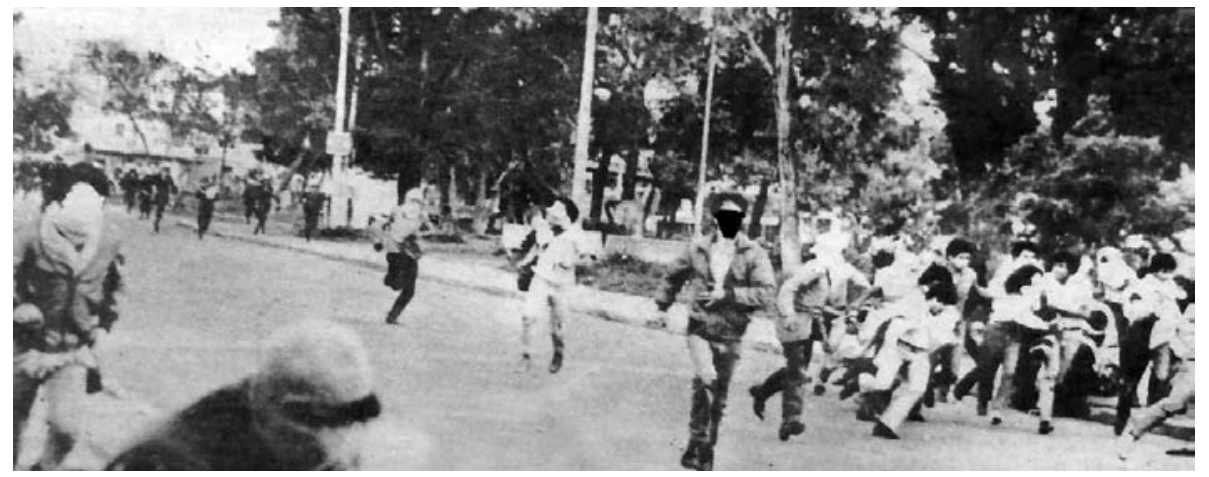

Fuente: El Bogotano, 17 de mayo de 1984.

Por otro lado, el periódico El Espacio (17 de mayo de 1984) señaló que «en el allanamiento a residencias universitarias y a varias facultades fueron detenidos 58 estudiantes y herido de gravedad con impacto de bala Raúl Martínez Gómez, quien quedó recluido en el Hospital de La Hortúa». Sobre este caso en particular, no fue encontrado un seguimiento y hasta ahora esta es la única información disponible.

\section{Conclusiones}

Dentro de lo expuesto en el presente trabajo, se han podido identificar por lo menos tres formas de negacionismo: (1) lo manifestado por el general Vargas Villegas, que niega o intenta encubrir algunos hechos documentados por otras fuentes como el ingreso de la Policía o la destrucción de edificios y bienes de la universidad; (2) lo expresado por Rafael Santos Calderón, quien, pese a la información que circuló en prensa de la época, que exponía casos de heridos y posibles muertos y desaparecidos, desconoció los hechos y las denuncias, con base en la incertidumbre 
que definió el contexto; y (3) lo expresado por Fernando Sánchez Torres, quien manifiesta un negacionismo que selectivamente condena y expone un tipo de violencia, en un recorte que le permite desconocer las afectaciones y violencias ocurridas sobre los y las universitarias.

No obstante, la escasa documentación sobre estos discursos públicos indica que no ha sido la circulación masiva de los mismos la que ha puesto lo ocurrido en las sombras. El silencio y el olvido que se imponen en este caso tienen que ver más con la falta de interés institucional por esclarecer los hechos violentos, aun cuando la gravedad de los mismos circuló de forma pública y contemporánea. Estos factores, entre otros, han derivado en que lo ocurrido en esas jornadas esté aún por esclarecerse, a fin de que se pueda garantizar plenamente el derecho de quienes se consideran víctimas o sobrevivientes a conocer la verdad, y a que las experiencias dolorosas que se relacionan con estos puedan ser reconocidas y valoradas públicamente.

Por otro lado, a partir de la información presentada, se puede identificar que las posturas que desestiman o niegan las denuncias de los estudiantes ocultan o no consideran una serie de hechos que dejan ver una intención de letalidad en la acción de los uniformados, cuando no pareciera que la justifican, como en el citado caso del artículo de Rafael Santos Calderón (El Tiempo, 19 de mayo de 1984). Frente a esto, la denuncia del caso de William Mayorga y los testimonios que presentó la Publicación 16 de mayo; lo publicado en la revista Cromos respecto al estudiante que recibió disparos en el rostro; el caso descrito por $\mathrm{El}$ Espacio (17 de mayo de 1984), en el que se señala que el estudiante Raúl Martínez Gómez fue herido de bala o aquel de El Bogotano (17 de mayo de 1984) que narra cómo otro estudiante recibió un disparo en el estómago y luego murió, además de la denuncia publicada por este mismo medio sobre seis estudiantes presuntamente desaparecidos, son descripciones de los hechos que cuentan un tipo de violencia ejercido por la fuerza pública, susceptible de ser pensado como un caso de victimización contra el estudiantado de la Universidad Nacional con 
pretensiones de letalidad. Cómo se señaló antes, en estos casos la escasa información disponible ha impedido el contraste de fuentes y la confirmación de algunas denuncias, no obstante, lo presentado es suficiente como para ser pensado a manera de indicios en el marco de un acontecimiento que merece ser esclarecido.

Finalmente, estimo que lo aquí expuesto debe ser considerado con atención, especialmente, cuando, a partir de 2019, ha entrado en funcionamiento el SIVJRNR, la CEV y el convenio celebrado entre esta entidad y la Universidad Nacional, pues su trabajo implica una ventana de oportunidad hasta ahora inexistente para comprender y dimensionar las consecuencias del conflicto armado en las universidades. Esto debe entenderse como parte del proceso de construcción de acuerdos ético políticos en los que todas las víctimas y los dolores importen, para que, a pesar de las posiciones empecinadas en la guerra, en un futuro, ojalá no tan lejano, la sociedad colombiana pueda desacostumbrarse a la presencia de la muerte y las luchas por la educación nunca más cobren la vida de nadie.

\section{Bibliografía}

\section{Archivo del Movimiento Estudiantil}

Asamblea UN (1988). Comunicado a la opinión pública [publicado con motivo de los acontecimientos violentos del 16 de mayo de 1988]. Fuente: Proyecto Archivos del Búho.

Coordinadora de Grupos de Trabajo de la Universidad Nacional, CGTUN (1985). Carta a los estudiantes. Fuente: Archivo Central un.

Frente Estudiantil Revolucionario, FER-Sinpermiso (1988). Curso de inducción [documento presentado a los estudiantes de primer semestre]. Fuente: Proyecto Archivos del Búho.

Periódico Indi-gestión (1990). Fuente: Proyecto Archivos del Búho.

Publicación 16 de mayo, P16M (1984). Fuente: Proyecto Archivos del Búho. 


\section{Entrevistas}

Higuera, Luis (2018). En: 16 de mayo. Entrevistadores: Luis Fernando Gómez Alba y Juan Sebastián Flórez Herrera. Corto documental producido en el marco del programa Cinemateca Rodante de la Cinemática Distrital de Bogotá.

\section{Prensa}

El Bogotano (17 de mayo de 1984).

El Bogotano (18 de mayo de 1984).

El Bogotano (19 de mayo de 1984).

El Espectador (17 de mayo de 1984).

El Espacio (17 de mayo de 1984).

El Tiempo (19 de mayo de 1984).

Revista Cromos (17 de mayo de 1984).

Revista Semana (1. ${ }^{\circ}$ de noviembre de 1982). Edición del 1 de noviembre.

Revista Semana (1984). Edición del 19 de mayo.

Sánchez Torres, Fernando (18 de mayo de 2003). En honor a la verdad. UN Periódico.

\section{Institucionales}

Consejo Superior Universitario de la Universidad Nacional de Colombia. Resolución 185 de 1976. Consejo Superior Universitario de la Universidad Nacional de Colombia. Acuerdo 100 de 1984.

Universidad Nacional de Colombia (1983). Boletín de Estadística UN. Bogotá: Oficina de Planeación.

Universidad Nacional de Colombia (1985). Diagnóstico para una reforma académica y administrativa de la Universidad Nacional de Colombia. UN Bogotá: Rectoría. 
Universidad Nacional de Colombia (1996). Estadísticas e Indicadores de la Universidad Nacional 1970-1995. Bogotá: Oficina de Planeación. Dirección general: Rosa de Lima Gallo. Serie Informes.

\section{Académica}

Aponte, Juana (2006). El 16 de mayo como crimen de lesa humanidad (Trabajo de grado de pregrado del departamento de Ciencia Política). Bogotá: Universidad Nacional de Colombia.

Archila, Mauricio (2017). Memoria, verdad e historia oral. Revista Controversia, 209, pp. 21-39

Ortega, Francisco (2008). Rehabitar la cotidianidad. En: Das, Veena (Ed. Francisco A. Ortega), Sujetos de dolor, agentes de dignidad. Bogotá: Universidad Nacional de Colombia. Facultad de Ciencias Humanas, Pontificia Universidad Javeriana, Instituto Pensar, Lecturas CES.

Portelli, Alessandro (1989). Historia y Memoria: La Muerte De Luigi Trastulli. Historia y Fuente Oral, no. 1, 1989, pp. 5-32. JSTOR, www.jstor.org/ stable/27753227.

Sandoval, Beatriz y Pinzón, Magaly (1992). Descripción y análisis de los grupos políticos que han hecho presencia en la Universidad Nacional en el periodo 1985 y 1991 (tesis de pregrado del departamento de Trabajo Social). Bogotá: Universidad Nacional de Colombia.

Salamanca, Sebastián (2007). Ciudad blanca, ciudad fuego. En: Crónicas barriales. Bogotá: Biblioteca Luis Ángel Arango. 